\title{
Artesunate-enhanced apoptosis of human high-risk myelodysplastic cells induced by the DNA methyltransferase inhibitor decitabine
}

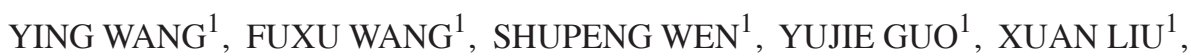 \\ XUEJUN ZHANG ${ }^{1}$ and LING PAN ${ }^{1,2}$ \\ ${ }^{1}$ Department of Hematology, The Second Hospital of Hebei Medical University, Shijiazhuang, Hebei 050000; \\ ${ }^{2}$ Department of Hematology, West China Hospital, Sichuan University, Chengdu, Sichuan 610041, P.R. China
}

Received February 27, 2014; Accepted November 12, 2014

DOI: 10.3892/ol.2015.3121

\begin{abstract}
The present study aimed to investigate whether artesunate (ART) could enhance the rate of apoptosis induced by decitabine (DAC) in the high-risk myelodysplastic syndrome (MDS) SKM-1 cell line, and examine the potential underlying mechanisms. The cytotoxicity and effect upon the apoptosis of ART and DAC in the SKM-1 cells was detected using the cell counting kit-8 assay and flow cytometry, respectively. The SKM-1 protein expression levels of activated caspase-3, -9 and -8 , cleaved poly(ADP-ribose) polymerase and apoptosis-inducing factor (AIF) were measured by western blotting. The laser confocal microscope analysis revealed AIF transfer to the nucleus. The growth inhibition and apoptosis rates of the ART- and DAC-treated SKM-1 cells were significantly increased compared with those of the single agent-treated SKM-1 cells $(\mathrm{P}<0.05)$. In addition, ART and DAC induced caspase-dependent apoptosis, while ART, but not DAC, induced caspase-independent apoptosis via AIF transfer from the mitochondria to the nucleus. In addition, ART-DAC-induced cell death was not attenuated by the caspase-3/7 inhibitor, Ac-DEVD-CHO. The results of the present study suggested that the ART-DAC combination exhibited increased effectiveness compared with the single-agent therapy, in vitro. The ART-DAC combined therapy not only activated a caspase-dependent apoptotic pathway, but also a caspase-independent mitochondrial pathway.
\end{abstract}

\section{Introduction}

Myelodysplastic syndromes (MDSs) are a group of life-threatening malignancies that primarily afflict the elderly population.

Correspondence to: Dr Ying Wang or Dr Ling Pan, Department of Hematology, The Second Hospital of Hebei Medical University, 215 West Heping Road, Shijiazhuang, Hebei 050000, P.R. China E-mail: wangying730@126.com

E-mail: panlingxueye1@126.com

Key words: artesunate, decitabine, apoptosis-inducing factor, caspase, myelodysplastic syndrome
The treatment of elderly patients with intensive chemotherapy is associated with high rates of treatment-related morbidity and mortality. The hypermethylation of $\mathrm{CpG}$ islands within promoter regions, and the resultant silencing of tumor-associated genes, are significant events involved in the pathogenesis of MDS (1). Previous studies have revealed that the DNA methyltransferase (DNMT) 1 and 3A enzymes contribute to aberrant methylation in MDS $(2,3)$. Therefore, DNMTs have become potential targets for the treatment of MDS (4-8). Two DNMT-targeted therapies, azacitidine (AZA) and decitabine [5-aza-2-deoxycytidine (DAC)], have been approved for clinical use by the Food and Drug administration (FDA) $(1,7)$. However, the direct cytotoxic effects upon the hematopoietic system caused by the approved DNMT inhibitors (DNMTIs), in addition to their chemical instability, has prompted the development of alternative therapeutic DNMTIs. The cytidine analogue, zebularine, is a third nucleoside DNMTI that has been developed. The mode of action of zebularine is similar to that of DAC, however, zebularine has an extended half-life compared with AZA and DAC, and can be selectively incorporated into malignant, rather than normal cells. The limitation to the development of zebularine is that higher concentrations of the drug are required to achieve comparable demethylation results to those observed following treatment with AZA and DAC (9-11).

In addition to identifying novel nucleoside DNMTIs, significant efforts have been made to develop non-nucleoside-targeted therapies that can directly inhibit individual DNMTs. However, the antisense oligonucleotide-targeting DNMTI, MG98, demonstrated inconsistent knock-down of DMNT mRNA in solid and hematopoietic tumors during phase I clinical trials, which was believed to be the result of inefficient intracellular MG98 uptake (12). At present, treatment with DAC alone has not demonstrated any additional significant survival benefits for high-risk MDS patients compared with traditional best supportive care (11). However, the use of DAC has been proposed as a low-intensity therapeutic approach for elderly patients with high-risk MDS and MDS/acute myeloid leukemia (AML), who are considered unfit to undergo aggressive chemotherapy (13). Due to a lack of specificity, and its incorporation into DNA during DNA 
synthesis, DAC is able to induce DNA damage, mutagenesis, cytotoxicity and hypomethylation. Therefore, low-dose DAC regimens have been investigated in a number of clinical trials and have been shown to be effective. The results of the clinical trials demonstrated reduced levels of DAC-induced toxicity in elderly patients who were unsuitable for high-intensity induction chemotherapy and consecutive allogeneic hematopoietic stem cell transplantation (HSCT) (14). In addition, it has been revealed that low doses of DAC induce significant anti-MDS effects, a safe toxicity profile and a complete remission rate of $21-39 \%$ (14). However, primary and secondary resistance to low-dose DAC-based regimens is emerging as a significant clinical issue. The survival rate at relapse following an initial response to treatment is poor. One study identified that primary resistance to treatment resulted from the abnormal active metabolism of DAC, and that secondary resistance was due to the unbalanced activation of downstream pathways, such as the nuclear factor- $\kappa \mathrm{B}(\mathrm{NF}-\kappa \mathrm{B})$ and phosphatidylinositol 3-phosphate/AKT pathways, which limited the clinical effect of DAC (15).

Artesunate (ART) (Fig. 1), a safe and effective antimalarial drug, is a semi-synthetic derivative of the drug artemisinin, and is extracted from the Chinese herb Artemisia annua. Previous studies have demonstrated that ART inhibits the growth of a number of carcinoma cell lines, and therefore, may be a potential novel candidate for the treatment of cancer $(16,17)$. In the present study, the MDS SKM-1 cell line was co-treated with ART and DAC. This regimen was designed based on the knowledge that cell survival signal transduction pathways, namely the phosphatidylinositol 3-kinase (PI3K)-C2 $\alpha / \mathrm{AKT} 1$ and RELA/NF- $\kappa \mathrm{B}$ pathways, are involved in the secondary resistance to DAC, and that high-risk MDS has the tendency to transform towards AML (18). A previous study indicated that ART conferred anticancer and chemosensitive effects by downregulating the activity of the PI3K/AKT and NF- $\mathrm{BB}$ signaling pathways (19). At present, the positive effect of ART upon the rate of DAC-induced apoptosis and growth inhibition within high-risk MDS cells is yet to be elucidated. However, ART-DAC combined therapy may present an alternative strategy for the treatment of cancer. In the present study, the efficacy of ART in sensitizing high-risk human MDS cells to $\mathrm{DAC}$, and the intracellular signaling mechanisms that underlie ART-enhanced DAC-induced apoptosis and growth inhibition, were investigated.

\section{Materials and methods}

Chemicals and reagents. ART (Guilin Pharmaceutical Co., Ltd., Shanghai, China) was dissolved in dimethyl sulfoxide (DMSO) at a concentration of $20 \mathrm{mg} / \mathrm{ml}$ and kept as a stock solution at $-20^{\circ} \mathrm{C}$. The caspase $3 / 7$ inhibitor, Ac-DEVD-CHO (Sigma-Aldrich Shanghai Trading Co., Ltd., Shanghai, China), was dissolved in DMSO at a concentration of $100 \mathrm{mM}$. The final concentration of DMSO was kept below $0.1 \%$ throughout the study. DAC (Sigma-Aldrich Shanghai Trading Co., Ltd.) was dissolved in $0.01 \mathrm{M}$ phosphate-buffered saline (PBS) at a concentration of $5 \mathrm{mg} / \mathrm{ml}$. Cell counting kit-8 (CCK-8) was purchased from Dojindo Laboratories (Kumamoto, Japan), the RPMI 1640 medium and fetal bovine serum (FBS) were from GIBCO (Carlsbad, CA, USA) and the Annexin V-fluorescein isothiocyanate (FITC) kit was from Bioseal Biotechnology Co., Ltd. (Beijing, China). The P1250 total protein extraction kit was obtained from Applygen Technologies Inc. (Beijing, China), the BCA protein assay kit was from the Beijing Biosynthesis Biotechnology Co., Ltd. (Beijing, China), the goat anti-rabbit immunoglobulin $\mathrm{G}(\mathrm{IgG})$ horseradish peroxidase (HRP)-conjugated secondary antibody (dilution, 1:100) was from Santa Cruz Biotechnology, Inc. (Santa Cruz, CA, USA) and the western chemiluminescent HRP substrate was from Millipore Corporation (Billerica, MA, USA). The primary polyclonal rabbit anti-human antibodies (dilution, 1:1,000), specific to caspase-3 (cat. no. 9664), -8 (cat. no. 4790) and -9 (cat. no. 9502) and apoptosis-inducing factor (AIF) (cat. no. 4642), were obtained from Cell Signaling Technology (Shanghai) Biological Reagents Co., Ltd., (Shanghai, China), while the primary poly(ADP-ribose) polymerase (PARP) monoclonal rabbit anti-human antibody (dilution, 1:1,000) was purchased from Epitomics, Inc. (cat. no. E78, Zhejiang, China) and the rabbit anti-human GADPH antibody was from Santa Cruz Biotechnology, Inc. (cat. no. SC-25778; dilution, 1:1,000). Hoechst 33342, dissolved in $0.01 \mathrm{M}$ PBS at a concentration of $10 \mathrm{mg} / \mathrm{ml}$, and propidium iodide (PI) were purchased from Sigma-Aldrich Shanghai Trading Co., Ltd.

Cell culture and treatment. The SKM-1 cells, derived from a patient with high-risk MDS, were purchased from the Japanese Collection of Research Bioresources Cell Bank (Osaka, Japan). The cells were cultured in a flask containing RPMI 1640 medium supplemented with 10\% FBS, 100 U/ml penicillin and $100 \mu \mathrm{g} / \mathrm{ml}$ streptomycin at $37^{\circ} \mathrm{C}$ in a humidified $5 \% \mathrm{CO}_{2}$ incubator. In total, two MDS patients, treated at The Second Hospital of Hebei Medical University (Shijiazhuang, China), were included in the present study. Subsequent to obtaining informed consent, mononuclear cells were isolated from the heparinized bone marrow of the patients by Ficoll-Hypaque density centrifugation (600 x g, $20 \mathrm{~min})$, and cultured in RPMI 1640 containing 10\% FBS, $2 \mathrm{mM}$ L-glutamine, $100 \mathrm{U} / \mathrm{ml}$ penicillin and $100 \mu \mathrm{g} / \mathrm{ml}$ streptomycin at $37^{\circ} \mathrm{C}$ in a humidified $5 \% \mathrm{CO}_{2}$ incubator. This study was approved by the Medical Ethics Committee of the Second Hospital of Hebei Medical University.

Cell growth inhibition assay. The cell viability, following treatment with or without DAC and ART, was measured using CCK-8. The SKM-1 and fresh bone marrow mononuclear cells (BMMNCs) were seeded into a 96-well plate at a density of $1 \times 10^{4} / 160 \mu \mathrm{l}$ per well. Subsequent to a $4-\mathrm{h}$ incubation, medium containing $10 \mu \mathrm{l} \mathrm{DAC}$, at a final concentration of $1,4,8,80$ or $1,600 \mu \mathrm{mol} / 1$, was added to each well and incubated for a further $30 \mathrm{~min}$. Next, medium containing $10 \mu \mathrm{l}$ ART, at a final concentration of $1,5,10$ or $100 \mu \mathrm{mol} / 1$, was added to certain wells, and further incubated for $24 \mathrm{~h}$. In total, $20 \mu \mathrm{l}$ WST-8 solution was added to each well and incubated at $37^{\circ} \mathrm{C}$ for $4 \mathrm{~h}$. Optical density (OD) was calculated from $450 \mathrm{~nm}$ absorption $\left(\mathrm{A}_{450}\right)$ values using an automated enzyme-linked immunosorbent assay reader (PerkinElmer, Shanghai, China). The cell viability was determined from the absorbance of soluble formazan dye generated by living cells. The cell growth inhibition ratio was calculated as follows: 

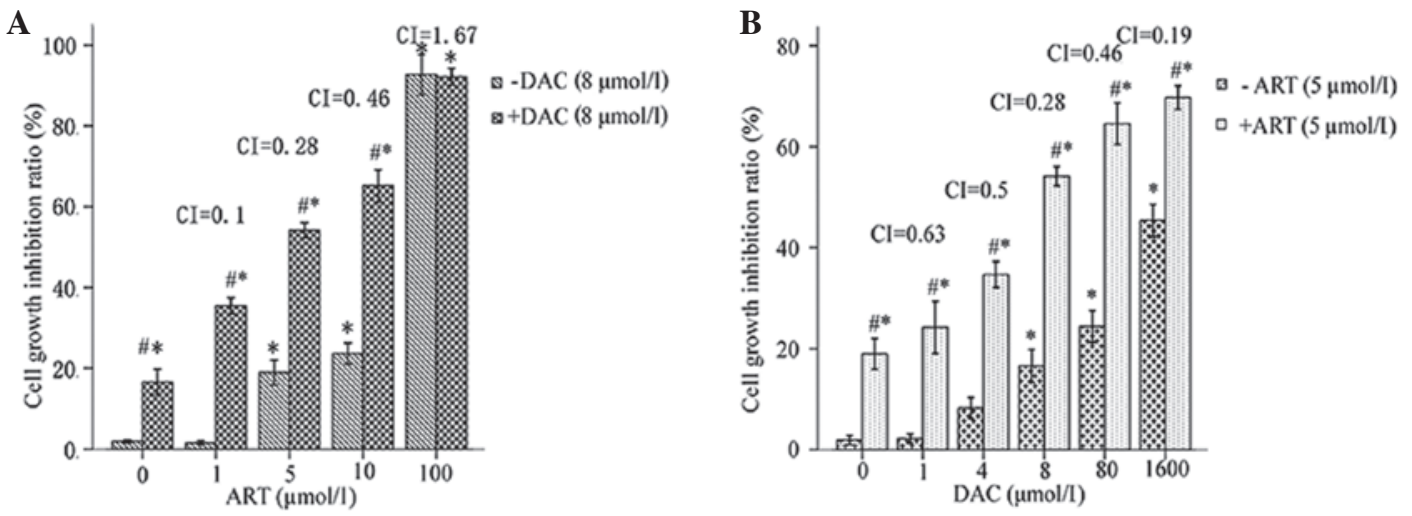

Figure 1. Cell growth inhibition ratio of SKM-1 cells incubated with different concentrations of artesunate (ART) and/or decitabine (DAC) for 24 h. (A) Cells treated with varying concentrations of ART, with or without $8 \mu \mathrm{mol} / 1 \mathrm{DAC}$. (B) Cells treated with varying concentrations of DAC, with or without $5 \mu$ mol/1 ART. ${ }^{\text {"P }}<0.05$ ART+DAC treated group vs. ART-treated; and ${ }^{*} \mathrm{P}<0.05$ ART or ART+DAC treated vs. the control without ART or DAC treated. CI, combination index.

Inhibition ratio $=1-\mathrm{A}_{450}$ of experimental well / $\mathrm{A}_{450}$ of blank control well $\mathrm{x} 100$. In total, each assay was repeated at least three times. The combined effects were evaluated using the combination index (CI) isobologram. The general equation for $\mathrm{CI}$ is as follows: $\mathrm{CI}=(\mathrm{D})_{1} /(\mathrm{Dx})_{1}+(\mathrm{D})_{2} /(\mathrm{Dx})_{2}$, where $\mathrm{CI}<1$ indicates synergism, $\mathrm{CI}=1$ indicates an additive effect and $\mathrm{CI}>1$ indicates antagonism (20).

Cell apoptosis assay. The quantitative analysis of cellular apoptosis was performed using Annexin V-FITC/PI double staining. In total, $1.0 \times 10^{5} \mathrm{SKM}-1$ or BMMN cells were planted in 24-well plates and exposed to $8 \mu \mathrm{mol} / 1 \mathrm{DAC}$ and/or $5 \mu \mathrm{mol} / 1$ ART for $24 \mathrm{~h}$. Next, flow cytometry, with fluorescence channels 1 and 2, was used to calculate the percentage of apoptotic cells (BD FACS Canto ${ }^{\mathrm{TM}}$ II; BD Biosciences, Franklin Lakes, NJ, USA). The apoptotic morphology of SKM-1 cells was analyzed by staining the cells with $10 \mu \mathrm{g} / \mathrm{ml}$ of the fluorescent DNA-binding dye, Hoechst 33342 , and $20 \mu \mathrm{g} / \mathrm{ml}$ PI at $37^{\circ} \mathrm{C}$ for $15 \mathrm{~min}$ after culturing in 6-well plates in the aforementioned manner. Apoptotic cells were identified by the presence of condensed and fragmented nuclei using fluorescence microscopy (BX51, Olympus, Tokyo, Japan).

Western blot analysis. The SKM-1 cells, treated as aforementioned, were harvested, washed twice with ice-cold PBS and lysed in lysis buffer containing $50 \mathrm{mM}$ HEPES (pH 7.4), $1 \%$ Triton X-100, $2 \mathrm{mM}$ sodium orthovanadate, $100 \mathrm{mM}$ sodium fluoride, $1 \mathrm{mM}$ EDTA, $1 \mathrm{mM}$ EGTA and $1 \mathrm{mM}$ phenylmethanesulfonylfluoride. The lysis buffer was supplemented with proteinase inhibitors; $10 \mu \mathrm{g} / \mathrm{ml}$ aprotinin, $10 \mu \mathrm{g} / \mathrm{ml}$ leupeptin and $100 \mu \mathrm{g} / \mathrm{ml}$ pepstatin, and incubated at $4^{\circ} \mathrm{C}$ for $1 \mathrm{~h}$. Subsequent to a 15 -min $9,500 \mathrm{x}$ g centrifugation at $4^{\circ} \mathrm{C}$, the cellular protein concentration was determined using the BCA protein assay kit (Beijing Biosynthesis Biotechnology Co., Ltd.). Equal quantities of total proteins were separated using SDS-PAGE, and then transferred to a polyvinylidene fluoride membrane (Sino-American Biotechnology Co. Ltd., Shanghai, China). The proteins, caspase- $3,-8$ and -9 , and AIF, were detected using the indicated primary antibodies and the HRP-conjugated secondary antibody. The signal was detected using the ChemiDoc XRS+ system with enhanced chemiluminescence, and analyzed by the Image Lab software (Bio-Rad Laboratories, Inc., Hercules, CA, USA).

Immunofluorescence assay. The immunofluorescence staining was performed using paraformaldehyde-fixed cell slices from SKM-1 cells exposed to either Ac-DEVD-CHO or DMSO. After blocking against non-specific binding using goat serum, the cells were exposed to the primary antibodies against caspase- $3,-8$ and -9 , and AIF, followed by secondary antibody conjugation to the FITC (1:100). The cells were counterstained using Hoechst 33342 (Beijing Biosea Biotechnology Co., Ltd., Beijing, China), and then analyzed in images captured by confocal fluorescence microscopy (TE-2000, Nikon, Tokyo, Japan).

Statistical analysis. All data were analyzed using SPSS v.13.0 software (SPSS, Inc., Chicago, USA) and expressed as the mean \pm standard error of the mean from three independent experiments performed in triplicate. All data were normally distributed, and analyzed further by analysis of variance and least significant difference as a post hoc test. $\mathrm{P}<0.05$ was considered to indicate a statistically significant difference.

\section{Results}

ART enhances DAC-induced cell growth inhibition. In the preliminary experiment, SKM-1 cellular cytotoxicity was determined following a 24-h treatment with 0-100 $\mu \mathrm{mol} / \mathrm{l}$ ART. A concentration range of 1-5 $\mu \mathrm{M}$ ART demonstrated only slight toxicity in the SKM-1 cells, as the cell viability was $>80 \%$ following treatment (Fig. 1A). In addition, the cytotoxicity of the combination ART-DAC treatment was investigated. The results revealed that ART induced a significant positive synergist effect with DAC upon cell growth inhibition (Fig. 1A). Therefore, ART at a concentration of $5 \mu \mathrm{mol} / 1$ was selected for further studies. A 24-h treatment with DAC at concentrations of $1-8 \mu \mathrm{mol} / 1$ induced $<20 \%(16.56 \pm 3.21 \%)$ of SKM-1 cells to apoptose. This indicated that the SKM-1 cells were resistant to low-dose DAC-mediated growth inhibition following $24 \mathrm{~h}$ of treatment (Fig. 1B). However, the combination of $5 \mu \mathrm{mol} / 1$ ART with DAC significantly increased the percentage of dead cells by $14.15-40.62 \%$, depending on the 

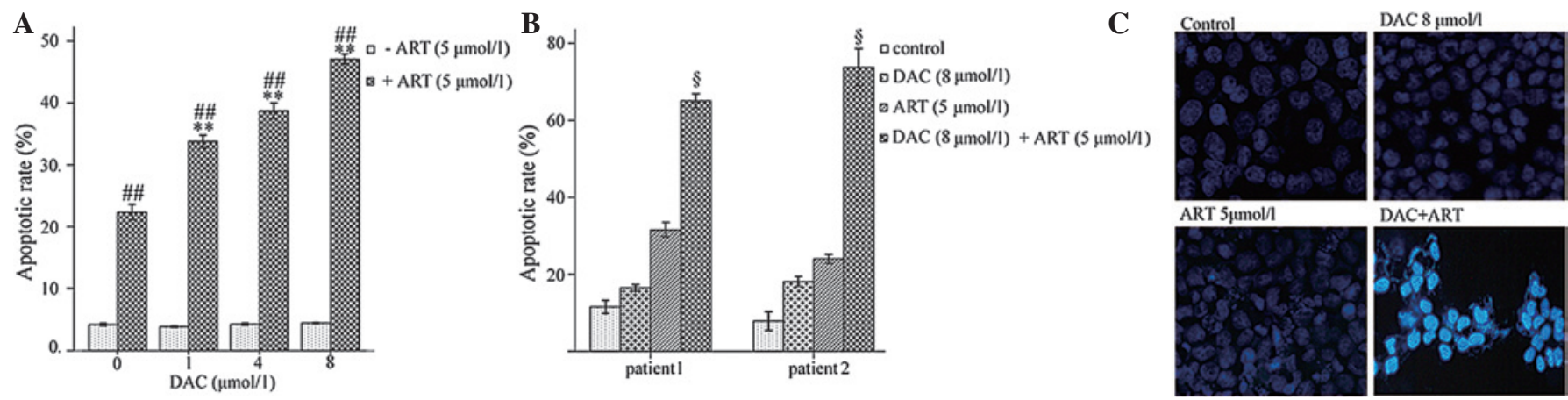

Figure 2. (A) Rate of apoptosis in SKM-1 cells exposed to different concentrations of decitabine (DAC) for 24 h, with or without $5 \mu$ mol/1 artesunate (ART). (B) The rate of apoptosis in patient bone marrow mononuclear cells exposed to $8 \mu \mathrm{mol} / 1$ DAC for $24 \mathrm{~h}$, with or without $5 \mu$ mol/1 ART. (C) Subsequent to a 24-incubation, the condensed and fragmented nuclei of DAC- and/or ART-treated SKM-1 cells were visible following staining with Hoechst 33342/propidium iodide. ${ }^{* *} \mathrm{P}<0.01 \mathrm{ART}+\mathrm{DAC}$ treated group vs. DAC-treated; ${ }^{\# \#} \mathrm{P}<0.005 \mathrm{ART}+\mathrm{DAC}$ treated group vs. control (without ART or DAC ); and ${ }^{\S} \mathrm{P}<0.005$ vs. the other groups.

concentration of DAC used (1-8 $\mu \mathrm{mol} / \mathrm{l})$ (Fig. 1B). In addition, the enhancement of DAC-induced cell death by ART was dose-dependent, as revealed by a Spearman's @ correlation coefficient of $0.712(\mathrm{P}=0.009)$. Therefore, a concentration of $5 \mu \mathrm{mol} / 1$ ART combined with $8 \mu \mathrm{mol} / 1 \mathrm{DAC}$ was selected for further studies.

ART-DAC combination induces an increased rate of apoptosis of MDS cells. The ART-DAC combination treatment significantly increased the rate of apoptosis in the SKM-1 cells and the BMMNCs from high-risk MDS patients compared with DAC or ART alone ( $\mathrm{P}<0.005$; Fig. $2 \mathrm{~A}$ and $\mathrm{B})$. The apoptotic cells, which exhibited bright-blue staining and fragmented nuclei following incubation with Hoechst 33342/PI, are shown in Fig. 2C. The data suggests that compared with the single-agent treatment, the ART-DAC combination markedly enhanced the rate of apoptosis in the SKM-1 cell line and in the primary BMMNCs obtained from the high-risk MDS patients.

ART-DAC combination enhances the rate of apoptosis of MDS cells through activation of caspase-3-dependent and -independent death signals. In order to identify the molecular mechanisms by which the combined ART-DAC treatment enhanced the rate of apoptosis in the MDS cells, western blot assays were used to analyze the activation of the caspases, and the cleavage of PARP in SKM-1 cells that had been incubated with $8 \mu \mathrm{mol} / 1 \mathrm{DAC}$ and/or $5 \mu \mathrm{mol} / 1 \mathrm{ART}$ for $12 \mathrm{~h}$. The results revealed that the combination ART-DAC treatment significantly increased the activation of caspase-3 and -9, and the cleavage of PARP compared with DAC or ART alone (Fig. 3A). To confirm the occurrence of caspase-dependent apoptosis in high-risk MDS cells, the SKM-1 cells were incubated with $20 \mu \mathrm{mol} / 1$ Ac-DEVD-CHO, a caspase-3 and -7 inhibitor, for $1 \mathrm{~h}$ prior to treatment with ART and/or DAC. Following incubation, it was revealed that Ac-DEVD-CHO abrogated DAC-induced, but not ART-induced, apoptosis (Fig. 3Ba). Therefore, the results indicated that DAC and ART induce caspase-dependent and -independent apoptotic pathways in SKM-1 cells, respectively.

To further investigate the ART-induced caspase-independent apoptotic pathway, the SKM-1 cells were incubated with $8 \mu \mathrm{mol} / 1$
DAC and/or $5 \mu \mathrm{mol} / 1 \mathrm{ART}$ for $6 \mathrm{~h}$ following a 1-h pre-treatment with either DMSO $(1: 1,000)$ or $20 \mu \mathrm{mol} / 1$ Ac-DEVD-CHO. Following incubation, laser confocal microscopy analysis revealed that ART, but not DAC, induced AIF release from the mitochondria to the cytosol and into the nucleus. Furthermore, pre-treatment with AC-DEVD-CHO was unable to inhibit the release and transfer of AIF (Fig. 3Bb). The results indicated that ART, but not DAC, induced caspase-independent apoptosis via AIF transfer from the mitochondria to the nucleus. Furthermore, ART and DAC were able to induce caspase-dependent apoptosis, but the active caspase- 3 and cleaved PARP, induced by DAC or ART, were not observable $12 \mathrm{~h}$ later. The cells treated with the ART-DAC combination demonstrated the most significant activity of caspase-3 and cleaved PARP (Fig. 3C). The activation of two apoptotic signaling pathways supported the hypothesis that ART and DAC induce apoptosis via two distinct signaling cascades. The combined ART-DAC treatment significantly enhanced the rate of apoptosis in the SKM-1 cells compared with ART or DAC alone.

\section{Discussion}

With respect to drug resistance, MDS is considered a significantly challenging disease to treat. This is due, in part, to the heterogeneity of the malignancy and the failure of current therapies to sustain durable remissions. In clinical practice, cases of relapsed/refractory MDS prove difficult to treat. Therefore, novel therapeutic strategies that target MDS are required. At present, the only approved curative treatment for patients with MDS is HSCT. However, this approach is associated with high treatment-related morbidity and mortality. Other FDA approved treatments, namely DAC and AZA, have been used in low-intensity MDS therapeutic regimens. However, due to untargeted DNA damage and mutagenesis, and the occurrence of primary and secondary resistance, DAC and AZA have demonstrated limited clinical responses $(12,15)$. The future of MDS-targeted therapies may therefore require combinations of novel drugs. The original use of ART was as an effective anti-malaria agent, however, the drug has also demonstrated potent effects against certain malignant diseases. Previous preclinical data has revealed that ART is active in vitro and in vivo in cases of multiple myeloma and leukemia $(21,22)$. The 


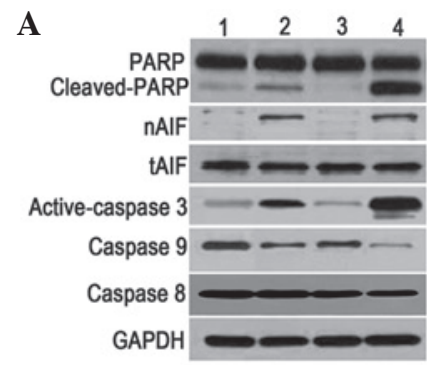

B

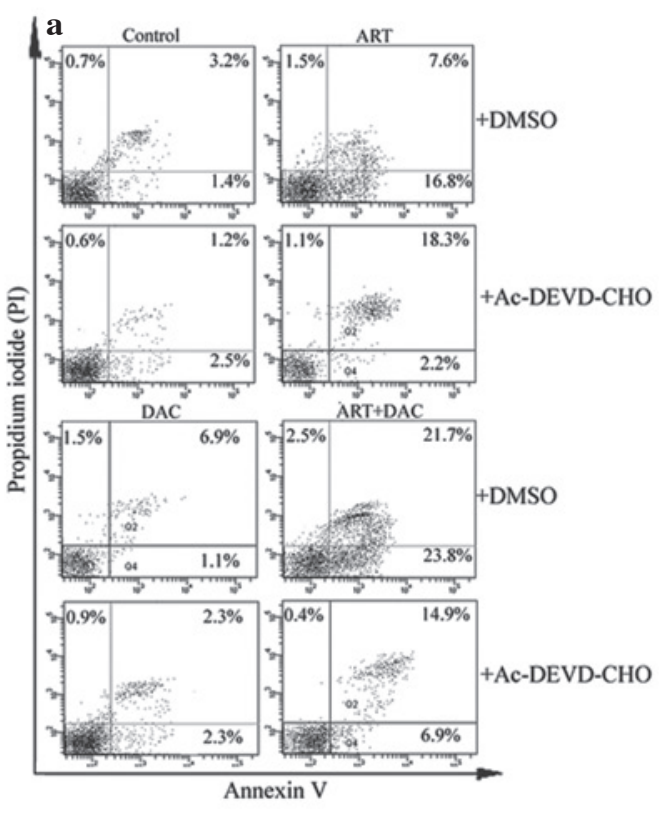

b
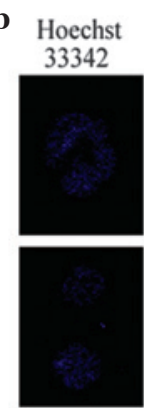

Control
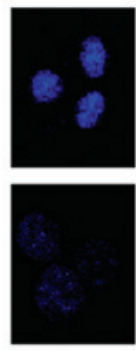

$\overline{D A C}$

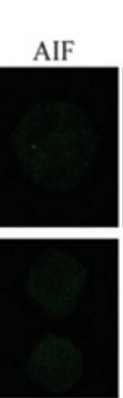

A

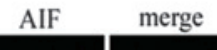

Hoechst
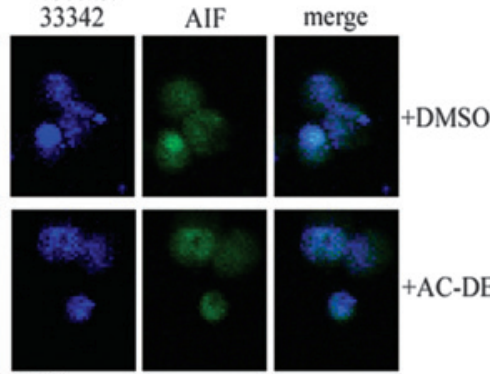

+AC-DEVD-CHO
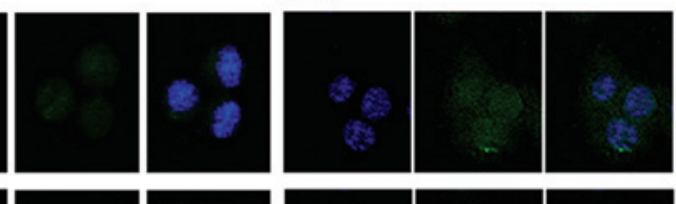

+DMSO
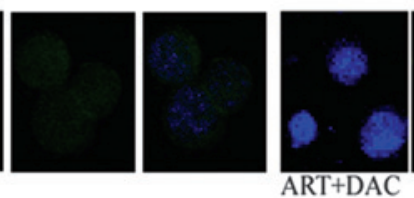

C

Control $5 \mu M$ ART $8 \mu M$ DAC ART+DAC

$\begin{array}{llllllllllll}3 & 6 & 12 & 3 & 6 & 12 & 3 & 6 & 12 & 3 & 6 & 12\end{array}$
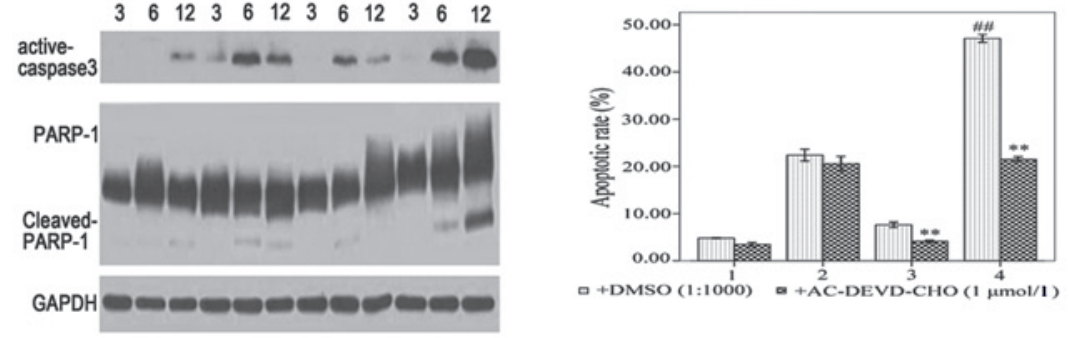

Figure 3. (A) SKM-1 cells were treated with $8 \mu \mathrm{mol} / 1$ decitabine (DAC) or $0.1 \%$ dimethylsulfoxide (DMSO) for 30 min, and then further incubated with $5 \mu \mathrm{mol} / 1$ artesunate (ART) for $12 \mathrm{~h}$. Whole cell lysates or nuclear proteins were analyzed by western blotting for the apoptotic proteins, caspase- $9,-8$, and-3, poly(ADP-ribose) polymerase-1 (PARP-1) and apoptosis-inducing factor (AIF), with GAPDH as the loading control. Lane 1, control group; lane 2, ART group; lane 3, DAC group; and lane 4, DAC and ART group. (B) (a) SKM-1 cells were treated with $8 \mu \mathrm{mol} / 1 \mathrm{DAC}$ or $0.1 \%$ DMSO for 30 min, and then further incubated with $5 \mu \mathrm{mol} / 1$ ART for $12 \mathrm{~h}$. The Annexin V/propidium iodide staining revealed that compared with single-agent treatment, the combination of ART and DAC induced an increased rate of apoptosis. The caspase-3/7 inhibitor, Ac-DEVD-CHO, abrogated apoptosis induced by DAC, but not ART. (B) (b) SKM-1 cells were treated with $8 \mu \mathrm{mol} / 1 \mathrm{DAC}$ or $0.1 \%$ DMSO for $30 \mathrm{~min}$, and then further incubated with $5 \mu \mathrm{mol} / 1 \mathrm{ART}$ for $6 \mathrm{~h}$. Laser confocal microscope analysis revealed that ART, but not DAC, induced AIF transfer to the nucleus. (C) SKM-1 cells were treated with DAC and/or ART for 3, 6 or $12 \mathrm{~h}$. Western blot analysis revealed that DAC and ART induced caspase-dependent apoptosis. The presence of active caspase-3 and cleaved PARP, induced by DAC or ART alone, was not observed after $12 \mathrm{~h}$. However, the combined ART-DAC treatment induced a notable activation of caspase-3 and cleaved PARP, which was observed even after $12 \mathrm{~h} .{ }^{* *} \mathrm{P}<0.005$ compared with the control group treated with DMSO; and ${ }^{\# \#} \mathrm{P}<0.005$ compared with the other groups.

present study identified that ART activated caspase-dependent and caspase-independent mitochondrial pathways. Furthermore, the study revealed that the AIF-mediated caspase-independent pathway was the primary cascade responsible for the initiation of apoptosis in the SKM-1 cells. The molecular mechanism by which DAC initiated apoptosis in SKM-1 cells was revealed to be associated with the activation of caspases- 9 and -3 , and with the cleavage of PARP (Fig. 3). In addition, the effect of the ART-DAC combination treatment upon high-risk MDS cells was analyzed in vitro in order to identify the underlying therapeutic mechanisms. These mechanisms may then provide the basis for future clinical trials that examine cases of relapsed or refractory MDS. The results revealed that the combination of ART and DAC inhibited the growth of the SKM-1 cell line in vitro (Fig. 1). 
Furthermore, it was identified that compared with single-agent treatment, the combined treatment significantly enhanced the rate of apoptosis in the SKM-1 and primary high-risk MDS cells in vitro (Fig. $2 \mathrm{~A}$ and $\mathrm{C}$ ). In addition, the combination of ART and DAC significantly enhanced the effects of apoptosis in the cells obtained from patients with MDS (Fig. 2B). The western blot analysis confirmed that compared with ART or DAC alone, the combined ART-DAC treatment activated caspase- 9 and -3 , and cleaved PARP more potently. The pre-treatment of cells with the caspase-3 and -7 inhibitor, Ac-DEVD-CHO, abrogated DAC-induced, but not ART-induced, apoptosis (Fig. 3A and B). Furthermore, following a 12-h incubation with ART, but not DAC, it was revealed that AIF was released from the mitochondria and was transited to the nucleus (Fig. 3C). The ART-induced apoptosis of the SKM-1 cells was identified to be primarily caspase-independent, and therefore, not completely inhibited by the caspase-3 and -7 inhibitor, Ac-DEVD-CHO. The results of the present study support the hypothesis that the combination of ART and DAC induces apoptosis in MDS cells via two distinct signaling cascades. This additive response is consistent with the observation that the ART-DAC combination treatment activated a caspase-dependent and -independent mitochondrial pathway.

In conclusion, the results of the present study indicated that compared with single-agent therapy, the combination of ART and DAC exhibited an increased efficacy against MDS cells in vitro. The combined treatment may confer increased antitumor activity and overcome specific cases of cellular resistance and/or identify novel anti-apoptotic mechanisms. Considering the results from the present study, future studies may be performed that address these issues and investigate the therapeutic effects of combined treatment approaches in patients with relapsed or refractory MDS. Additional studies are required to affirm these results, and to provide an improved understanding of the mechanisms, interactions and cellular targets that are involved in the signaling pathways targeted by drugs such as ART and DAC.

\section{Acknowledgements}

The present study was supported by the Natural Science Foundation of Hebei Province (no. C2008001097). The present study was supported by the Medical Science Research Project of Hebei Province(no. 20130186). The authors would like to thank the Department of Cardiology, Second Hospital of Hebei Medical University for providing assistance with the western blot analysis.

\section{References}

1. Fenaux P, Mufti GJ, Hellstrom-Lindberg E, et al: Efficacy of azacitidine compared with that of conventional care regimens in the treatment of higher-risk myelodysplastic syndromes: a randomised, open-label, phase III study. Lancet Oncol 10: 223-232, 2009.
2. Stankov K, Bogdanovic G, Kojic V, et al: Expression analysis of genes involved in epigenetic regulation and apoptosis in human malignant haematopoietic cell lines treated with 5-azacytidine. J BUON 16: 116-122, 2011.

3. Saunthararajah Y, Triozzi P, Rini B, et al: p53-independent, normal stem cell sparing epigenetic differentiation therapy for myeloid and other malignancies. Semin Oncol 39: 97-108, 2012.

4. Lin J, Yao DM, Qian J, et al: Recurrent DNMT3A R882 mutations in Chinese patients with acute myeloid leukemia and myelodysplastic syndrome. PLoS One 6: e26906, 2011.

5. Walter MJ, Ding L, Shen D, et al: Recurrent DNMT3A mutations in patients with myelodysplastic syndromes. Leukemia 25: 1153-1158, 2011.

6. Shih AH, Abdel-Wahab O, Patel JP and Levine RL: The role of mutations in epigenetic regulators in myeloid malignancies. Nat Rev Cancer 12: 599-612, 2012.

7. Faderl S, Garcia-Manero G, Jabbour E, et al: A randomized study of 2 dose levels of intravenous clofarabine in the treatment of patients with higher-risk myelodysplastic syndrome. Cancer 118: 722-728, 2012.

8. Piekarz RL and Bates SE: Epigenetic modifiers: basic understanding and clinical development. Clin Cancer Res 15: 3918-3926, 2009.

9. Cheng JC, Matsen CB, Gonzales FA, et al: Inhibition of DNA methylation and reactivation of silenced genes by zebularine. J Natl Cancer Inst 95: 399-409, 2003.

10. Robak T: New nucleoside analogs for patients with hematological malignancies. Expert Opin Investig Drugs 20: 343-359, 2011.

11. Flotho C, Claus R, Batz C, et al: The DNA methyltransferase inhibitors azacitidine, decitabine and zebularine exert differential effects on cancer gene expression in acute myeloid leukemia cells. Leukemia 23: 1019-1028, 2009.

12. Götze K, Platzbecker U, Giagounidis A, et al: Azacitidine for treatment of patients with myelodysplastic syndromes (MDS): practical recommendations of the German MDS Study Group. Ann Hematol 89: 841-850, 2010.

13. Klisovic RB, Stock W, Cataland S, et al: A phase I biological study of MG98, an oligodeoxynucleotide antisense to DNA methyltransferase 1 , in patients with high-risk myelodysplasia and acute myeloid leukemia. Clin Cancer Res 14: 2444-2449, 2008.

14. Yang X, Lay F, Han H and Jones PA: Targeting DNA methylation for epigenetic therapy. Trends Pharmacol Sci 31: 536-546, 2010.

15. Qin T, Castoro R, El Ahdab S, et al: Mechanisms of resistance to decitabine in the myelodysplastic syndrome. PLoS One 6: e23372, 2011.

16. Efferth T, Giaisi M, Merling A, Krammer PH and Li-Weber M: Artesunate induces ROS-mediated apoptosis in doxorubicin-resistant T leukemia cells. PLoS One 2: e693, 2007.

17. Mercer AE, Copple IM, Maggs JL, O'Neill PM and Park BK: The role of heme and the mitochondrion in the chemical and molecular mechanisms of mammalian cell death induced by the artemisinin antimalarials. J Biol Chem 286: 987-996, 2011.

18. Kondo A, Yamashita T, Tamura H, et al: Interferon-gamma and tumor necrosis factor-alpha induce an immunoinhibitory molecule, B7-H1, via nuclear factor-kappaB activation in blasts in myelodysplastic syndromes. Blood 116: 1124-1131, 2010.

19. Thanaketpaisarn O, Waiwut P, Sakurai H and Saiki I: Artesunate enhances TRAIL-induced apoptosis in human cervical carcinoma cells through inhibition of the NF- $\mathrm{KB}$ and PI3K/Akt signaling pathways. Int J Oncol 39: 279-285, 2011.

20. Chou TC and Talalay P: Analysis of combined drug effects: a new look at a very old problem. Trends Phamacol Sci 4: 450-454, 1983.

21. Chen H, Shi L, Yang X, Li S, Guo X and Pan L: Artesunate inhibiting angiogenesis induced by human myeloma RPMI8226 cells. Int J Hematol 92: 587-597, 2010.

22. Wang Y, Han Y, Yang Y, et al: Effect of interaction of magnetic nanoparticles of $\mathrm{Fe}_{3} \mathrm{O}_{4}$ and artesunate on apoptosis of $\mathrm{K} 562$ cells. Int J Nanomedicine 6: 1185-1192, 2011. 